\title{
ARTICLE OPEN Suppressing the ferroelectric switching barrier in hybrid improper ferroelectrics
}

\author{
Shutong Li (iD) and Turan Birol (iD ${ }^{1 凶}$
}

Integration of ferroelectric materials into novel technological applications requires low coercive field materials, and consequently, design strategies to reduce the ferroelectric switching barriers. In this first principles study, we show that biaxial strain, which has a strong effect on the ferroelectric ground states, can also be used to tune the switching barrier of hybrid improper ferroelectric Ruddlesden-Popper oxides. We identify the region of the strain-tolerance factor phase diagram where this intrinsic barrier is suppressed, and show that it can be explained in relation to strain-induced phase transitions to nonpolar phases.

npj Computational Materials (2020)6:168; https://doi.org/10.1038/s41524-020-00436-x

\section{INTRODUCTION}

Since the discovery of ferroelectricity in $\mathrm{BaTiO}_{3}$, perovskite oxides have been heavily studied and utilized in applications as ferroelectric materials. Versatility of the perovskite structure allows a large number of complex oxides to be synthesized, but among those, only a small fraction are ferroelectrics ${ }^{1}$. A major breakthrough in perovskite-related ferroelectrics is the discovery of hybrid improper ferroelectricity (HIF) as a materials design route in 2011, which led to an explosion in the predictions of novel ferroelectric oxides ${ }^{2}$. Among those, the list of examples that are experimentally verified includes $\mathrm{A}_{3} \mathrm{~B}_{2} \mathrm{O}_{7} \mathrm{HIFs}\left(\mathrm{Ca}, \mathrm{Sr}_{3}\right)_{3} \mathrm{Ti}_{2} \mathrm{O}_{7}{ }^{3},(\mathrm{Sr}$, $\mathrm{Ca})_{3} \mathrm{Sn}_{2} \mathrm{O}_{7}, \mathrm{Sr}_{3} \mathrm{Zr}_{2} \mathrm{O}_{7}{ }^{4-6}$, as well as a weak ferromagnetic $\left(\mathrm{Ca}_{0.69} \mathrm{Sr}_{0.46} \mathrm{~Tb}_{1.85} \mathrm{Fe}_{2} \mathrm{O}_{7}\right)^{7}$.

Despite the prediction of ferroelectricity and observation of a polar crystal structure in many compounds, experimentally observing the switching of polarization is challenging. For example, the original $\mathrm{HIF} \mathrm{Ca}_{3} \mathrm{Ti}_{2} \mathrm{O}_{7}$ was reported to have a polar structure 20 years before the idea of HIFs was introduced ${ }^{8}$, but the direct evidence of polarization switching was not observed until $2015^{3}$. The reason behind the absence of switching in these materials was initially believed to be large intrinsic coercive fields, or defects in the materials, which typically increase the coercive field ${ }^{9,10}$. The high experimental coercive field is not surprising, because the energy scale that needs to be overcome for switching is considered to be determined by the octahedral rotations, which often have an energy scale significantly higher than that of the ferroelectric distortions in typical perovskite oxides. Switching was observed in other HIF materials with coercive fields ranging from 120 to $200 \mathrm{kV}$ $\mathrm{cm}^{-13,4,6}$, and very recently, the smallest coercive field of $39 \mathrm{kV}$ $\mathrm{cm}^{-1}$ was observed in single crystals of $\mathrm{Sr}_{3} \mathrm{Sn}_{2} \mathrm{O}_{7}{ }^{11}$. Though these coercive fields are comparable to values suitable for integration to silicon chips $\left(E_{\mathrm{c}} \approx 50 \mathrm{kV} \mathrm{cm}^{-1}\right)$, applications such as high-power actuators and low-voltage logic and memory elements ask for ferroelectrics with robust polarizations that can be switched by a lower coercive field ${ }^{12-15}$. Ultra-low coercive fields as low as $5 \mathrm{kV}$ $\mathrm{cm}^{-1}$ were observed in pulsed laser deposition grown $\mathrm{Ca}_{3} \mathrm{Ti}_{2} \mathrm{O}_{7}$ thin films, but the reason behind this reduction (and whether it is an intrinsic or an extrinsic effect) is not clarified yet ${ }^{16}$.

Understanding the intrinsic mechanisms that affect the coercive field of HIF materials, and finding new design strategies to reduce these fields are important for their applications. In this paper, we illustrate that strain can be an effective means to achieve this. Epitaxial strain, obtained by growing thin films on latticemismatched substrates, has been used extensively as a way to tune the ferroelectric and dielectric properties of perovskites ${ }^{17,18}$. Both the octahedral rotations, and the proper ferroelectric order parameter are strongly coupled with the biaxial strain in most materials, and strain is shown to change the switching energy barrier of ferroelectrics as well ${ }^{19}$. HIFs are shown to undergo interesting structural phase transitions under strain as well ${ }^{20}$, but there is no detailed study of the switching behavior of HIFs under biaxial strain. The original study on $\mathrm{HIFs}^{2}$ showed that the lowest energy switching path and energy (which is correlated with the coercive field) is strain dependent, but the recent works that illustrate the richness of possible switching paths make it necessary to re-evaluate the polarization switching behavior of strained $\mathrm{HIFs}^{21,22}$.

In this study, we perform density functional theory (DFT) calculations on 13 different $\mathrm{A}_{3} \mathrm{~B}_{2} \mathrm{O}_{7}$ Ruddlesden-Popper compounds to map out the strain-tolerance factor phase diagram, and show that the strain-induced non-polar or anti-polar phases emerge in compounds with a finite range of tolerance factors. We then show, by performing nudged elastic band (NEB) calculations, that the intrinsic coherent polarization switching energy barrier decreases as the compounds get closer to phase boundaries by biaxial strain. This suppression of switching barrier is not always accompanied with a decrease in the polarization, which makes strain tuning of HIF Ruddlesden-Poppers a viable tool to obtain low coercive field ferroelectrics with a robust polarization. We also show that the tensile and compressive strains favor different switching pathways, which can be intuitively understood in terms of which octahedral rotations or tilts are favored by strain.

This paper is organized as follows: We start by explaining the crystal structures and important normal modes. We then present and discuss the strain-tolerance factor phase diagram of HIF RPs. Then, we present the trends of the intrinsic switching barrier as a function of strain. We conclude with a brief summary and discussions.

\section{RESULTS}

Review of crystal structures

The $\mathrm{A}_{3} \mathrm{~B}_{2} \mathrm{O}_{7}$ compounds considered in this study are the $n=2$ members of the Ruddlesden-Popper series ${ }^{23,24}$. They can be

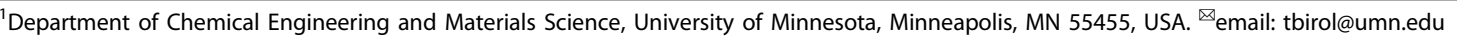




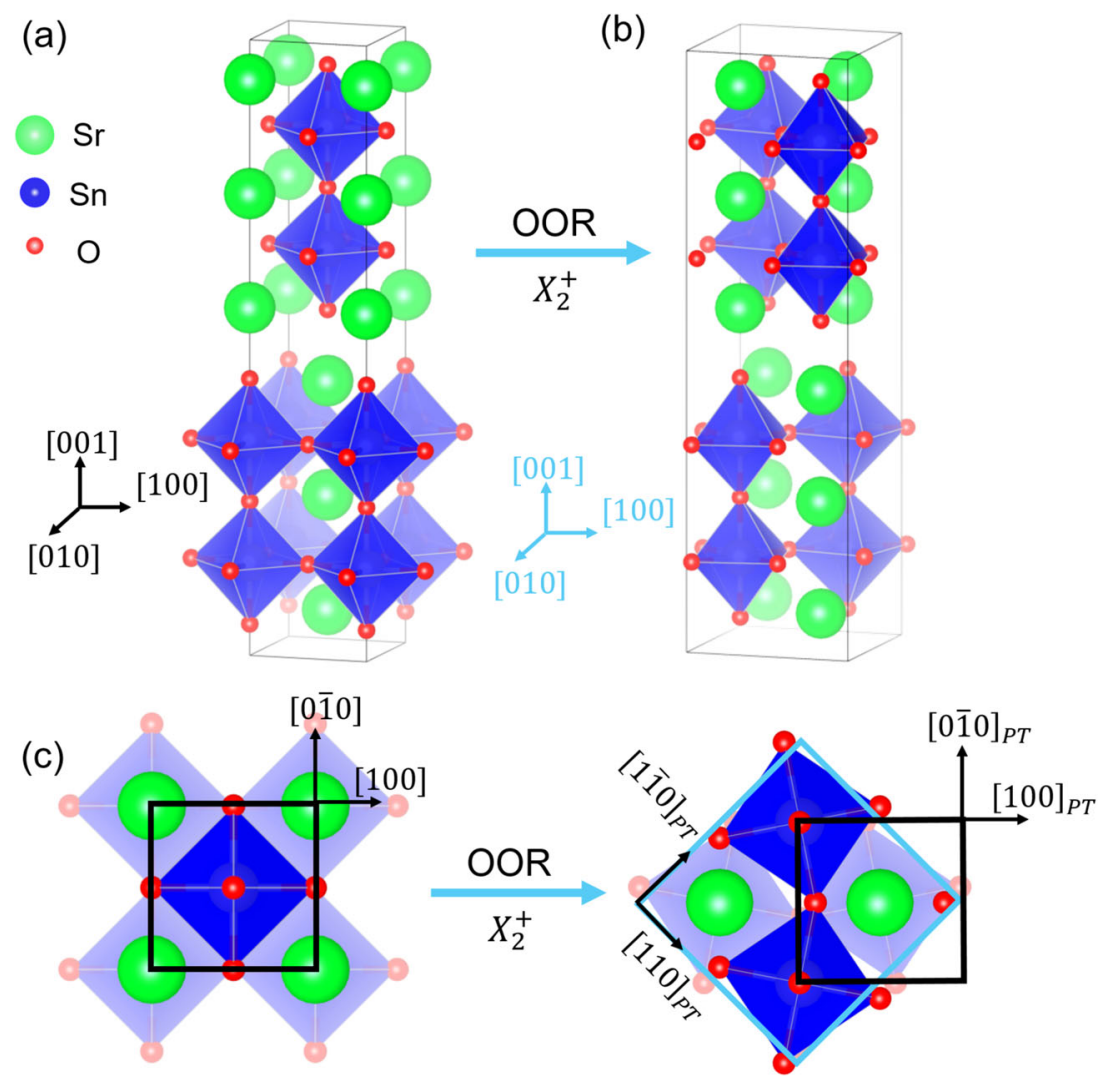

Fig. 1 The $\boldsymbol{n}=\mathbf{2}$ Ruddlesden-Popper structure. a The high symmetry body-centered-tetragonal phase $\left(14 / \mathrm{mmm}^{2}\right.$ of $\mathrm{A}_{3} \mathrm{~B}_{2} \mathrm{O}_{7} \mathrm{RP}-\mathrm{phase}$ perovskites. b Compounds with tolerance factor $<1$ develop octahedral rotation/tilt distortions, which are usually associated with normal modes at the $X$ point of the Brillouin zone. (The figure shows the $X_{2}^{+}$mode.) These distortions double the original unit cell and symmetry becomes orthorhombic. c Orientations of the crystal axes in the orthorhombic cell are different from those in the high-symmetry tetragonal cell. Throughout this paper, we use the axes of a pseudo-tetragonal cell (shown in black) that can be defined within the orthorhombic cell (shown in light blue).

considered as layered perovskites with an extra AO layer inserted after every two perovskite bi-layers (i.e. four atomic layers) along the [001] direction (Fig. 1a). The extra AO layers cause a shift by $(a / 2, a /$ $2,0)$ on the $a b$ plane, and hence the structure becomes bodycentered tetragonal with space group $14 / \mathrm{mmm}$ (\#139). This shift also breaks the connectivity of the oxygen octahedra, and the AO double layer is held together by mostly ionic bonds between the A-site cations and $\mathrm{O}$ anions. The resulting dimensional reduction has important consequences on the electronic structure and lattice response (for example, refs ${ }^{25-28}$ ). Apart from the dimensional effects, the different periodicity of the Ruddlesden-Popper phases along the layering direction (c-axis, or the [001] direction) leads to a smaller Brillouin zone than $\mathrm{ABO}_{3}$ perovskites. The equivalents of various structural instabilities that are at different points of the Brillouin zone in the $\mathrm{ABO}_{3}$ perovskites can fold back onto the same point in $\mathrm{A}_{3} \mathrm{~B}_{2} \mathrm{O}_{7}$ Ruddlesden-Poppers, which leads to interesting couplings between them as discussed below. (This point can be qualitatively understood in analogy to a subduction problem, where a zone boundary mode of the parent group corresponds to a zone center mode of the subgroup. For example, when the unitcell of a cubic perovskite is doubled along the [001] axis as a result of cation order, the spacegroup becomes $P 4 / \mathrm{mmm}$ and the zone boundary $X_{5}^{-}$mode splits into $\Gamma_{5}^{-} \oplus X_{2}^{-} \oplus X_{3}^{-}$, where $\Gamma_{5}^{-}$is polar. While there is no direct group-subgroup relationship between the Ruddlesden-Popper and perovskite structures, the $n=2$ Ruddlesden-Poppers have two perovskite blocks in their unit cells, and it is thus possible to recognize some phonon modes folded onto the $k_{z}=0$ plane).

By far the most common structural distortions that decrease the symmetry of oxide perovskites are the oxygen octahedral rotations: About $90 \%$ of all oxide perovskites have this type of distortions in their crystal structures, which reduce the symmetry of the parent $P m \overline{3} m$ phase ${ }^{29}$. These distortions can be described in terms of symmetry-adapted-modes, which can be classified by irreducible representations (irreps) of the parent spacegroup $P m \overline{3} m^{30}$. The phonon modes that correspond to these distortions are the $M$ point mode $M_{2}^{+}$, which is an in-phase rotation of octahedra around one axis, and the $R$ point mode $R_{5}^{-}$, which is an out-of-phase rotation of octahedra around one axis. The former is denoted by $\mathrm{a}^{\prime}+{ }^{\prime}$ ' superscript in the Glazer notation, such as $a^{0} a^{0} c^{+}$, and the latter is denoted by a '-' superscript, such as $a^{-} a^{-} a^{-}$. The most common rotation pattern that more than half of all oxide perovskites have is $a^{-} a^{-} c^{+}$, which leads to the space group Pnma (\#62) ${ }^{31}$. Another distortion that is often significant in the Pnma structure is the $X_{5}^{-}$out-of-phase A-site displacement. Unlike the $M_{2}^{+}$and $R_{5}^{-}$, the $X_{5}^{-}$often does not show up as an unstable phonon mode in the high symmetry $(P m \overline{3} m)$ phase. Rather, it is an improper order parameter, which attains a nonzero magnitude only because of a trilinear coupling in the Landau free energy

$\mathcal{F}_{\text {trilinear }}=\gamma M_{2}^{+} R_{5}^{-} X_{5}^{-}$.

The presence of $\mathcal{F}_{\text {trilinear }}$ in the free energy expansion, which is imposed by group theory, guarantees a nonzero $X_{5}^{-}$distortion whenever the octahedral rotations $M_{2}^{+}$and $R_{5}^{-}$are present, no matter the sign of the coupling $\gamma$.

Instabilities in the $\mathrm{A}_{3} \mathrm{~B}_{2} \mathrm{O}_{7}$ Ruddlesden-Poppers that are similar to the $M_{2}^{+}$and $R_{5}^{-}$normal modes in the $\mathrm{ABO}_{3}$ perovskites give rise to a wider range of different combinations and resultant symmetries. (For simplicity, we follow the convention to refer 
(a)

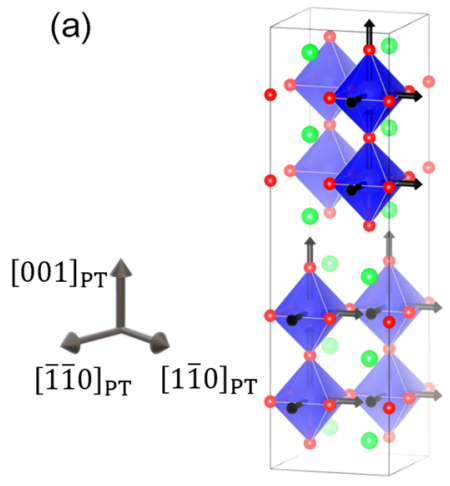

(c) (b)

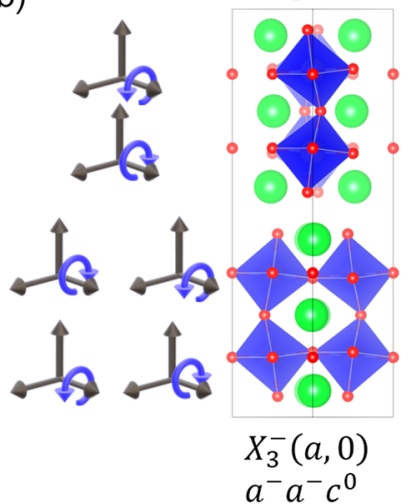

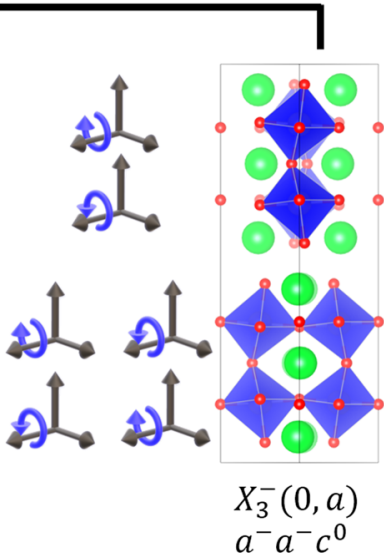
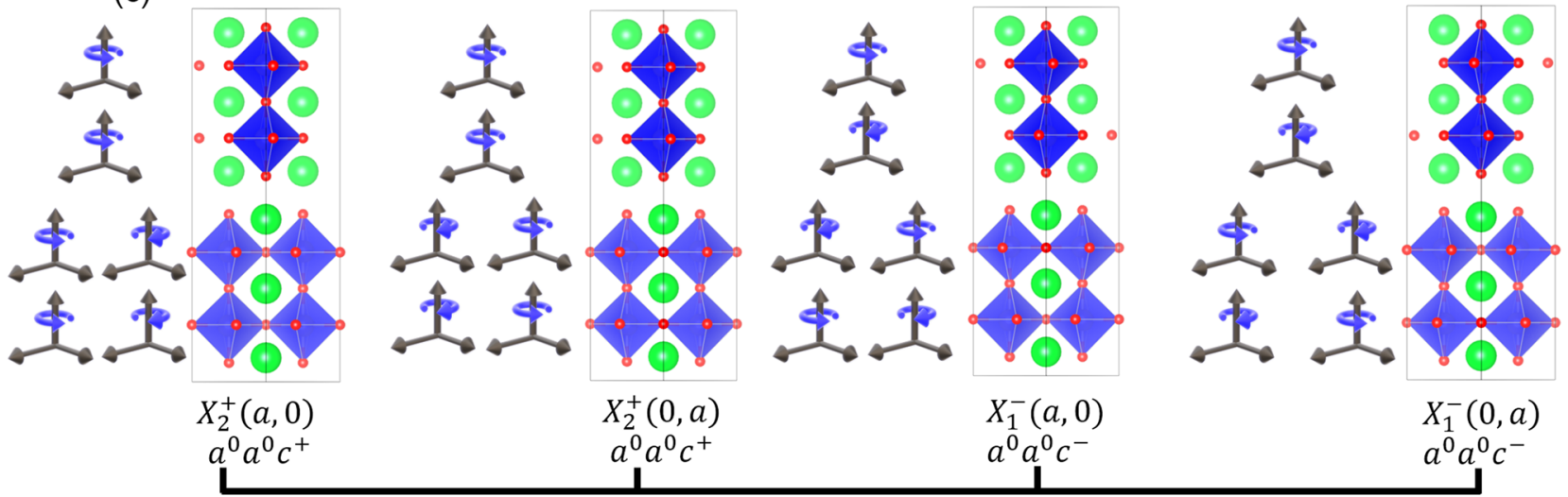

OOR

Fig. 2 The unstable modes at the $X$ point. a The undistorted structure in the orthorhombic supercell. The arrows on the octahedra are along the orthorhombic axes, and are parallel to the arrows in the other panels which denote the direction of octahedral rotations and tilts. b-d Distortion modes that correspond to different irreps. Both $X_{2}^{+}$and $X_{1}^{-}$modes are rotations around the $c$-axis. The $X_{2}^{+}$modes are in-phase while $X_{1}^{-}$modes are out-of-phase. The two components of the $X_{3}^{-}$mode are tilts around axes on $a b$ plane. While the words 'rotations' and 'tilts' are often used interchangeably in the literature, throughout this manuscript we consistently refer to rotations around the $c$-axis $\left(X_{2}^{+}\right.$and $\left.X_{1}^{-}\right)$as oxygen octahedral rotations (OOR), and rotations around the axes on the ab plane $\left(X_{3}^{-}\right)$as oxygen octahedral tilting (OOT).

octahedral rotations around the out-of-plane (c) axis as 'rotations' (OOR), and the rotations around the in-plane axes as 'tilts' (OOT).) One reason for this is that there is a new degree of freedom, since the body-centered primitive cell now contains two oxygen octahedra. Also, the double AO layers break the connectivity of oxygen octahedra, and hence the relative phase of neighboring octahedra on either side of the double layer is not fixed. As an example, we consider the modes relevant to the $A 2{ }_{1} a m$ phase observed in $\mathrm{Ca}_{3} \mathrm{Ti}_{2} \mathrm{O}_{7}$ and many other HIF Ruddlesden-Popper compounds in Fig. 2. In $\mathrm{ABO}_{3}$ perovskites, there are two possible rotation patterns around, for example, the $c$ axis: in-phase $\left(M_{2}^{+}\right.$, $\left.a^{0} a^{0} c^{+}\right)$or out-of-phase $\left(R_{5}^{-}, a^{0} a^{0} c^{-}\right)$. In the $\mathrm{A}_{3} \mathrm{~B}_{2} \mathrm{O}_{7}$, on the other hand, there are four possibilities: The $X_{2}^{+}$mode corresponds to an in-phase rotation of the two octahedra in one perovskite slab that consists of five atomic layers, and is the primitive unit cell. However, $X_{2}^{+}$is a two-dimensional irrep, and depending on its direction a particular pair of octahedra on either side of a double AO layer can have either in-phase or out-of-phase rotations, as shown on the left two panels of Fig. 2c. Similarly, the rotations that are out-of-phase within one perovskite slab transform as the twodimensional irrep $X_{1}^{-}$, as shown in the right panels of Fig. $2 \mathrm{c}$.

The most relevant octahedral rotation modes in $\mathrm{A}_{3} \mathrm{~B}_{2} \mathrm{O}_{7}$ all have the same wavevector: they correspond to $X$ point normal modes. This leads to a richer set of possibilities for the modes induced by trilinear couplings compared to $\mathrm{ABO}_{3}$ perovskites. In the trilinear coupling terms in $\mathrm{ABO}_{3}$ perovskites, an $M$ and an $R$ mode has to couple with an $X$ mode due to the translational symmetry. In $\mathrm{A}_{3} \mathrm{~B}_{2} \mathrm{O}_{7}$ compounds, on the other hand, the trilinear couplings that contain two separate $X$ modes can contain either an $M$ mode or a $\Gamma$ mode as the third mode. ( $M$ point is denoted as the $Z$ point in the convention of ref. ${ }^{32}$.) The reason is that there are two separate $X$ points on the Brillouin zone that are related to each other via a four-fold rotation, and depending on which pair of $X$ wavevectors are chosen, their sum can either give the $\Gamma$ or the $M$ wavevector. In Table 1, we list the possible trilinear couplings between two $X$ modes and a third mode in the $\mathrm{A}_{3} \mathrm{~B}_{2} \mathrm{O}_{7}$ structure, and in Fig. 3 , we display the polarization patterns of some of these structures.

HIF in the $A_{3} B_{2} O_{7}$ compounds emerges due to the trilinear coupling between $X_{2}^{+}$and $X_{3}^{-}$modes, which induces a polar displacement $\Gamma_{5}^{-}$. In the HIF structure with space group $A 2{ }_{1} a m$ (\#36), each $\mathrm{AO}$ layer has a polarization, which are in alternating directions within each perovskite slab, and hence cancel each other-but only partially. As a result, every perovskite slab between the double AO layers have a net dipole moment. These moments order in parallel and give rise to a macroscopic polarization (Fig. 3a). A different combination of the same $X$ modes can couple to the $M_{5}^{-}$mode, leading to anti-parallel slab dipoles, and hence to an anti-polar phase shown in Fig. 3b. (We refer to phases with nonzero dipole moments of each perovskite slab as either polar or antipolar.) Other combinations of the $X$ 
modes couple with different $M$ modes, such as $M_{5}^{+}$or $M_{2}^{+}$, and give rise to nonpolar phases, where the dipole moments of each atomic layer cancel each other within each perovskite slab between to double AO layers (Fig. $3 c$ and d). (We refer to phases where dipole moments of each slab are zero as 'nonpolar'.) Many of these phases are observed to emerge in various $\mathrm{A}_{3} \mathrm{~B}_{2} \mathrm{O}_{7}$ oxides under biaxial strain or equivalent doping, and are also shown to be important as intermediate states in the coherent switching of polarization $5,16,20-22,33$. This is in addition to single-tilt systems observed, for example, at finite temperature ${ }^{7}$. In the next subsection, we draw the strain-tolerance factor phase diagram of these compounds to identify regions where these antipolar and nonpolar multi-tilt phases emerge.

\section{Strain phase diagram}

Most - more than half- of oxide perovskites have a tolerance factor of $\tau<1$, and attain the space group Pnma at low temperatures ${ }^{29}$.

Table 1. List of structures that can be obtained by combining the unstable $X$ modes.

\begin{tabular}{llll}
\hline Irrep 1 & Irrep 2 & Coupled irreps & Space group \\
\hline$X_{1}^{-}(a, 0)$ & & & Aeaa (\#68) \\
$X_{2}^{+}(a, 0)$ & & & Aeam (\#64) \\
$X_{3}^{-}(a, 0)$ & & & Amam (\#63) \\
$X_{3}^{-}(a, a)$ & & $M_{2}^{+}(c)$ & $P 4_{2} / m n m(\# 136)$ \\
$X_{1}^{-}(a, 0)$ & $X_{3}^{-}(0, b)$ & $M_{5}^{+}(c, 0)$ & Pnab (\#60) \\
$X_{1}^{-}(a, 0)$ & $X_{3}^{-}(b, 0)$ & $\Gamma_{5}^{+}(c, 0)$ & $C / 2 c(\# 15)$ \\
$X_{1}^{-}(a, a)$ & $X_{3}^{-}(b, b)$ & $M_{1}^{+}(c), M_{2}^{+}(c), \Gamma_{4}^{+}(c), \Gamma_{5}^{+}(c, 0)$ & $C / 2 m(\# 12)$ \\
$X_{2}^{+}(a, 0)$ & $X_{3}^{-}(b, 0)$ & $\Gamma_{5}^{-}(c,-c)$ & A2 ${ }_{1} a m(\# 36)$ \\
$X_{2}^{+}(0, a)$ & $X_{3}^{-}(b, 0)$ & $M_{5}^{-}(0, c)$ & Pnam (\#62) \\
$X_{2}^{+}(a, a)$ & $X_{3}^{-}(b, b)$ & $M_{1}^{+}(c), M_{2}^{+}(c), \Gamma_{4}^{+}(c), \Gamma_{5}^{-}(c, 0)$ & $C 2 m(\# 38)$ \\
\hline
\end{tabular}

The trilinear couplings are obtained using the 'Invariants' tool in the Isotropy Software Suite ${ }^{65}$. While we performed DFT calculations for the energies of all of these phases, only the ones that are close to the lowest energy are shown in the plots.
The corresponding octahedral rotation pattern $a^{-} a^{-} c^{+}$is also common in $\mathrm{A}_{3} \mathrm{~B}_{2} \mathrm{O}_{7}$ Ruddlesden-Poppers, and gives rise to the polar space group $A 2{ }_{1} a m$ observed in HIFs. In addition to the polar phase, strain phase diagrams of these compounds often abound with transitions to nonpolar phases introduced in the preceding subsection. As an example, in Fig. 4a, we present the energy of three lowest energy structures for $\mathrm{Sr}_{3} \mathrm{Sn}_{2} \mathrm{O}_{7}$ as a function of biaxial strain $^{34}$. The zero temperature DFT calculations reproduce the experimentally observed room temperature phase $A 2{ }_{1} a m$ in the unstrained compound. Both tensile and compressive strain decrease the energy difference between this phase and the next lowest energy state, and there are phase transitions to nonpolar phases for strain $\gtrsim 2.5 \%$ on either direction. Similar strain-driven transitions have been predicted for $\mathrm{Sr}_{3} \mathrm{Zr}_{2} \mathrm{O}_{7}$ and $\mathrm{Ca}_{3} \mathrm{Ti}_{2} \mathrm{O}_{7} \mathrm{HIF}$ compounds previously, and the pattern of octahedral rotations often change under strain in the $\mathrm{ABO}_{3}$ compounds as well. $\mathrm{A}$ common trend in $\mathrm{A}^{2+} \mathrm{B}^{4+} \mathrm{O}_{3}$ perovskites is that tensile biaxial strain suppresses OOR around the out-of-plane axis, whereas compressive strain enhances it. $\mathrm{Sr}_{3} \mathrm{Sn}_{2} \mathrm{O}_{7}$ follows a similar trend: the transition under tensile strain is to the $P 4_{2} / \mathrm{mnm}$ phase, which has only $X_{3}^{-}$ tilts, whereas the transition under compressive strain is to the Aeaa phase, which has only the $X_{1}^{-}$rotations around the $c$ axis. The transition to these nonpolar phases is not a result of a continuous suppression of polarization by strain: the magnitudes of polarization in the $A 2_{1} a m$ phase on both phase boundaries are sizable, and is even enhanced under tensile strain, as shown in Fig. 4b.

In order to elucidate the behavior of different HIF compounds under strain, in Fig. 5 we map out the strain-tolerance factor phase diagram by considering 11 different $\mathrm{A}_{3} \mathrm{~B}_{2} \mathrm{O}_{7}$ compounds. (We do not include two compounds with larger tolerance factors, since they do not display any OOR or OOT. Most of these compounds have been studied from first principles before, but to the best of our knowledge, this is the first time that this information is compiled to display all compounds together. We consider a strain range of $\pm 4 \%$, which covers the experimentally feasible range. For most of the compounds with $\tau<1$ that we consider, the lowest energy unstrained structure is $A 2{ }_{1} a m$, which corresponds to the HIF phase. For $0.92 \lesssim \tau \lesssim 1$, nonpolar structures emerge under both tensile and compressive strain. We observe three different nonpolar structures: Pnab and $P 4_{2} / m n m$ under tensile strain, and $P n a b$ and Aeaa under compressive strain. They correspond to the following changes in the octahedral rotations and tilts:

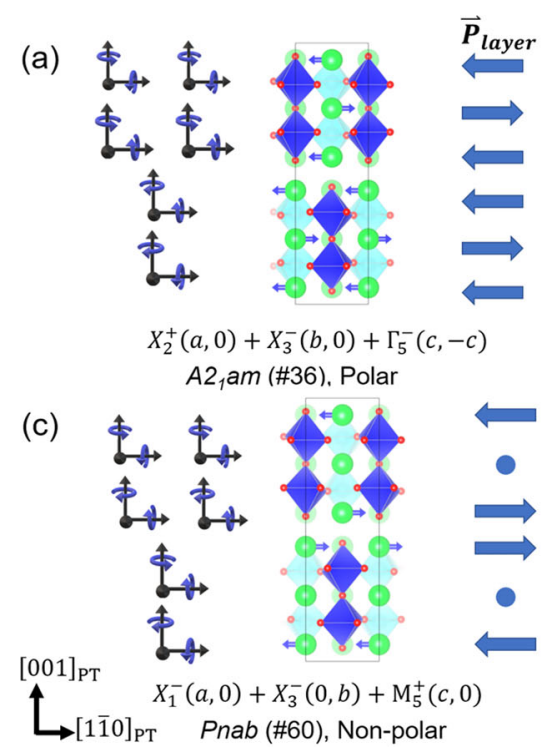

(b)

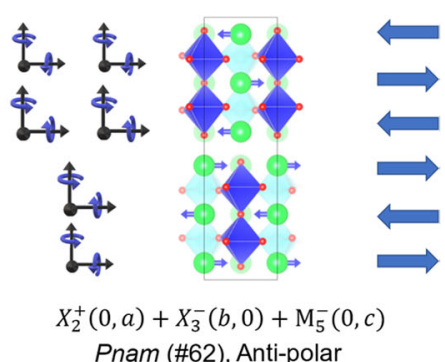

(d)

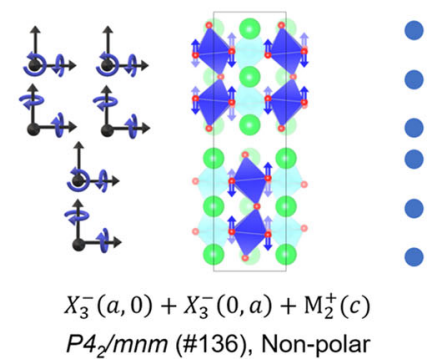

Fig. 3 Possible low-energy stable and metastable structures of RP-phase perovskites $A_{3} B_{2} O_{7}$ with more than one oxygen octahedral rotation modes. Analysis of these modes are presented in Table 1. 


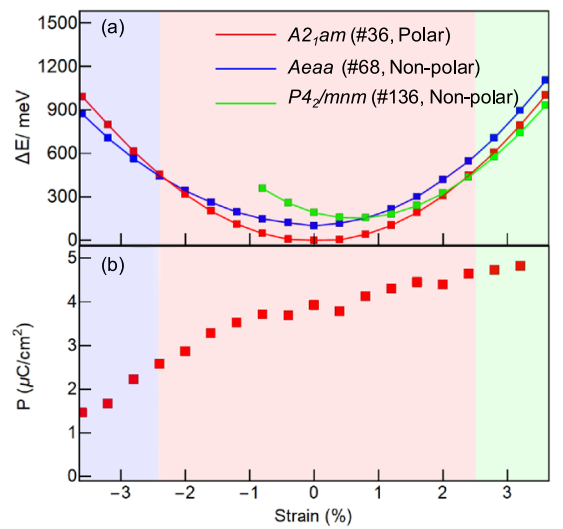

Fig. 4 Effect of strain on $\mathrm{Sr}_{3} \mathrm{Sn}_{2} \mathbf{O}_{7}$. a The energy of different metastable phases vary with biaxial strain. Transitions to nonpolar phases are observed on both tensile and compressive strain. $\mathbf{b}$ The polarization strength of the polar phase as a function of strain. The background colors indicate different ground state structures.

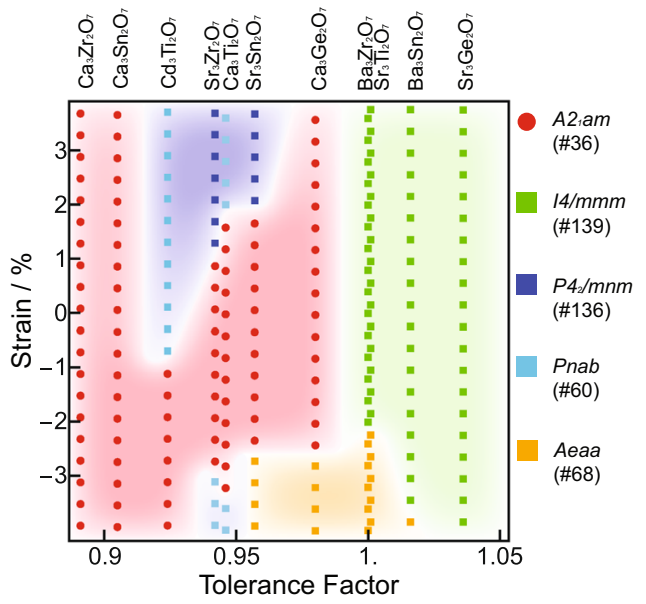

Fig. 5 Phase diagram of HIF $A_{3} B_{2} O_{7}$ compounds under biaxial strain. Red color represents ferroelectric (HIF) phase, the others are all non-polar structures. Results for $\mathrm{Ba}_{3} \mathrm{Ti}_{2} \mathrm{O}_{7}(t=1.06)$ and $\mathrm{Ba}_{3} \mathrm{Ge}_{2} \mathrm{O}_{7}$ $(t=1.10)$, which do not display any rotation or tilting, are not shown here. Proper ferroelectric phases of large tolerance factor compounds, such as the one in $\mathrm{Sr}_{3} \mathrm{Ti}_{2} \mathrm{O}_{7}$ under large tensile strain ${ }^{25,46}$, are not displayed either.

- Compressive strain-induced OOT suppression (leads to Aeaa): This is observed in $\mathrm{Sr}_{3} \mathrm{Sn}_{2} \mathrm{O}_{7}$ and $\mathrm{Ca}_{3} \mathrm{Ge}_{2} \mathrm{O}_{7}$. The OOT mode amplitude drops to zero and OOR mode phase changes under compressive strain as shown in Fig. $6 \mathrm{c}$, d.

- Tensile strain-induced OOR suppression (leads to $\mathrm{P4}_{2} / \mathrm{mnm}$ ): This is observed in $\mathrm{Sr}_{3} \mathrm{Zr}_{2} \mathrm{O}_{7}$ and $\mathrm{Sr}_{3} \mathrm{Sn}_{2} \mathrm{O}_{7}$. Similar to the first situation, but the OOR mode drops to zero under tensile strain instead of OOT mode, as shown in Fig. 6b, c.

- Tensile/compressive strain-induced OOR phase change (leads to Pnab): This is observed in $\mathrm{Ca}_{3} \mathrm{Ti}_{2} \mathrm{O}_{7}$ under both tensile and compressive strain, in $\mathrm{Sr}_{3} \mathrm{Zr}_{2} \mathrm{O}_{7}$ under compressive strain, or in $\mathrm{Cd}_{3} \mathrm{Ti}_{2} \mathrm{O}_{7}$ under small tensile as well as compressive strains (Fig. 6a, b). Amplitudes of both the OOR and OOT mode retain non-zero, but the in-phase OOR mode changes into out-ofphase manner. This structure is shown in Fig. 3b. The A-site cations around two interfaces move in the opposite direction, which cancels the polarization in bulk.

Some of these transitions are explained by local measures such as the global instability index (GII), which is known to predict the octahedral rotation patterns and angles in $\mathrm{ABO}_{3}$ perovskites successfully ${ }^{29,35}$. For example, the transition to $P 4_{2} / m n m$ in $\mathrm{Sr}_{3} \mathrm{Sn}_{2} \mathrm{O}_{7}$ is coincident with the strain value above which the GII of this phase is the smallest as shown in Supplementary Fig. 6 . However, the Gll by itself does not explain why the polar $A 2{ }_{1} a m$ structure is preferred over the Aeaa one, for these two phases have very similar GII values under compressive strain. It is possible that the interplay of Gll with the long-range Coulomb interaction (which is an important factor in stabilizing the polarization in proper ferroelectrics such as $\mathrm{BaTiO}_{3}{ }^{36}$ ) is responsible of the transition to the Aeaa phase.

The transition to a single-tilt system can be explained phenomenologically by the cross-term between OOR and OOT - a large OOR might suppress OOT and vice versa. All compounds in the $A 2{ }_{1} a m$ follow the same aforementioned trend as many $\mathrm{ABO}_{3}$ perovskites that compressive strain enhances OOR, whereas tensile strain enhances OOT (Fig. 6a-d) (for example, see refs ${ }^{20,37,38}$ ). This trend is likely the result of the strain reducing particular B-O bond lengths, which can be increased by the OOT or OOR distortions. The lowest order cross-term between the OOR and OOT in the free energy is $F \sim \beta R^{2} T^{2}$ (where we denote the amplitudes of rotations and tilts by $R$ and $T$, respectively). For fixed value of $R$, this term renormalizes the coefficient of the $T^{2}$ term $a T^{2}$ as $\sim\left(a+\beta R^{2}\right)$, and hence for large OOR $R^{2}>-\alpha / \beta$, the tilting instability is suppressed, and it becomes energetically favorable to have no tilts, as is the case in compressively strained $\mathrm{Sr}_{3} \mathrm{Sn}_{2} \mathrm{O}_{7}$ in the Aeaa phase.

A phenomenological explanation of the strain-induced transition to nonpolar Pnab structure requires not only the biquadratic terms between the OOR and the OOT modes, but also various trilinear terms that couple these modes to other antiferrodistortive displacements ${ }^{20}$. It is particularly interesting that in $\mathrm{Ca}_{3} \mathrm{Ti}_{2} \mathrm{O}_{7}$, this transition is re-entrant in the sense that it happens under both tensile and compressive strains. The Gll does not have an obvious trend that explains this transition, and the electrostatic interaction between the $\mathrm{O}$ ions on different layers is possibly important. We leave the microscopic explanation of this transition to a future study.

Strain tuning of the ferroelectric switching barrier

Enhanced susceptibilities near second-order phase transitions can be exploited to design materials with large responses, for example, magnetic permeability or dielectric constants. While no such enhancement of linear susceptibility is mandated near firstorder transitions, it is nevertheless possible to obtain large response near a first-order phase boundary if the external field can induce the transition. Examples of demonstrations of this approach include Terfenol, $\mathrm{Pb}(\mathrm{Zr}, \mathrm{Ti}) \mathrm{O}_{3}$, and $\mathrm{BiFeO}_{3}{ }^{39-41}$. The phase boundaries of structural transitions depend on strain very sensitively, and as a result, this approach is a promising means to enhance the response of materials via strain.

The question we focus on in this subsection is whether the ferroelectric polarization switching barrier is affected when strain is used to tune the materials to the vicinity of the polar-nonpolar phase transitions. In order to answer this question, we use the minimum energy barrier for coherent polarization switching as a proxy to the coercive electric field. While in an actual experiment defects, domain structure, as well as size and shape effects significantly alter the coercive field, trends of coherent switching barrier can be used as a first principles proxy to the trends of the coercive field ${ }^{42}$ as explicitly shown in $\mathrm{HfO}_{2}{ }^{19}$. (Finite element methods which take into account the domain structure provide much lower switching barriers ${ }^{43}$ ). In practice, the coherent switching field calculated from the first principles energy barrier by assuming that the dipole moment in every unit cell of an infinite crystal switches at the same time is a gross overestimate. As a result, we do not report the electric field required for switching, but instead report only the energy barriers. 
(a) $\mathrm{Ca}_{3} \mathrm{Ti}_{2} \mathrm{O}_{7}$

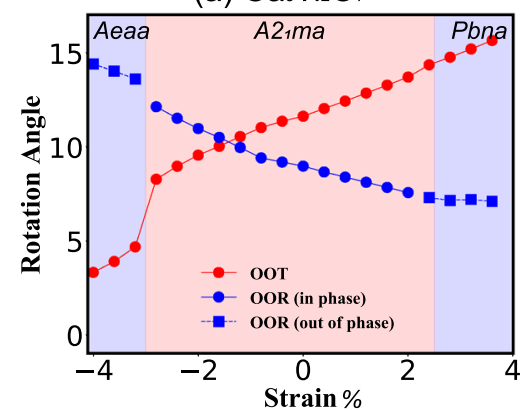

(c) $\mathrm{Sr}_{3} \mathrm{Sn}_{2} \mathrm{O}_{7}$

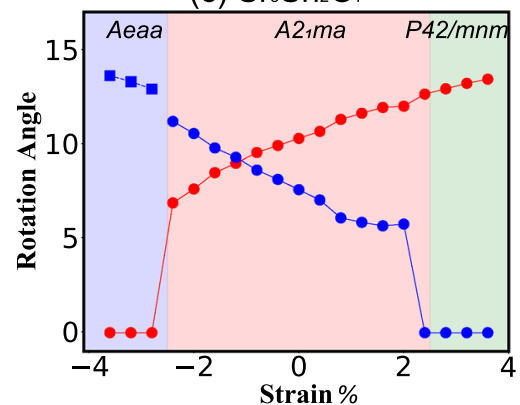

(b) $\mathrm{Sr}_{3} \mathrm{Zr}_{2} \mathrm{O}_{7}$

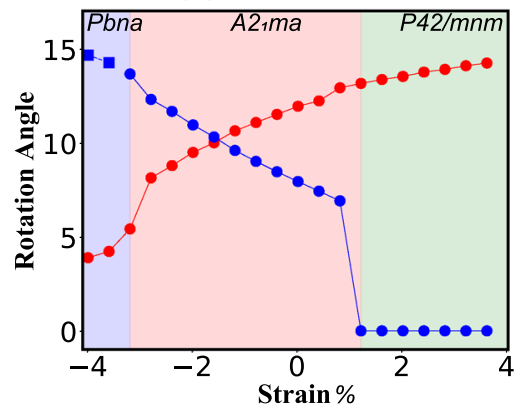

(d) $\mathrm{Ca}_{3} \mathrm{Ge}_{2} \mathrm{O}_{7}$

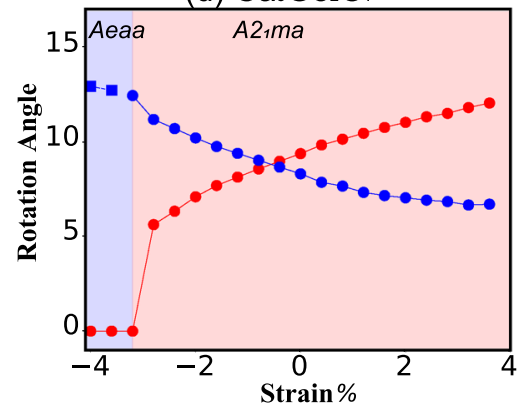

Fig. 6 Effect of strain on crystal structure. Rotation (OOR) and tilting (OOT) angles as a function of epitaxial strain in a $\mathrm{Ca}_{3} \mathrm{Ti}_{2} \mathrm{O}_{7}, \mathbf{b} \mathrm{Sr}_{3} \mathrm{Zr}_{2} \mathrm{O}_{7}, \mathbf{c}$ $\mathrm{Sr}_{3} \mathrm{Sn}_{2} \mathrm{O}_{7}$, and $\mathbf{d} \mathrm{Ca}_{3} \mathrm{Ge}_{2} \mathrm{O}_{7}$. Different colors represent different phases. Red regions are the ferroelectric phase.

Since in the hybrid improper ferroelectric $\mathrm{A}_{3} \mathrm{~B}_{2} \mathrm{O}_{7}$ compounds the polarization emerges as an improper order parameter through a trilinear coupling with rotation and tilting modes, switching one of these two modes is necessary to switch the polarization. It was recognized as early on as in the first HIF paper that this makes different switching pathways possible, and that the corresponding energy barriers can be tuned by strain ${ }^{2}$. Later, the work of Nowadnick and Fennie ${ }^{21}$ analyzed the possible roles of different switching mechanisms, and Munro et al. used the idea of distortion symmetry groups to identify other switching pathways ${ }^{22,44}$. Since then, the energetics of switching in various HIF compounds have been studied, for example in ref. ${ }^{45}$. However, to the best of our knowledge, a comparison of different compounds and their strain dependence have not been performed yet.

In Fig. 7a-d, we show four possible polarization switching pathways. We follow the convention of the distortion symmetry groups to name these pathways ${ }^{44}$. This process involves identifying not only the symmetry operations shared by all images on the pathway, but also those operations that reverse the distortion, which is the polarization in this case. The latter are referred to as distortion reversal symmetries, and are denoted by a ${ }^{* \prime \prime}$ superscript. For example, $P n^{*} a b$ means that each image along the switching path has two glide planes with translations along $a$ and $b$ axes; and the glide plane $n^{*}$ reverses the distortion. Three of the switching paths we consider $\left(P n^{*} a b, P b^{*} n m\right.$, and $\left.P n^{*} a m\right)$ have a similar name as their intermediate phase (up to the asterisks), because the spatial symmetry elements of the intermediate phase either remain unchanged or become reversal symmetry operation for other images. But this is not the case for $P n^{*} 2_{1}^{*} m$. All of the four are so-called two-step switching pathways, where there exists a local minimum of energy on the switching path, as seen from Fig. $8 \mathrm{a}$, and they are the lowest ones among such paths for the three compounds we considered. They each have distinct intermediate states, but the same initial and final states. Since the Ruddlesden-Popper structure consists of weakly bound perovskite blocks separated by an interface between two rocksalt AO layers, it is possible to consider supercells extended along the [001] direction, and polarization being switched in one perovskite block at a time. This, in principle, gives rise to an infinite number of different switching pathways, the barrier energy per formula unit can be arbitrarily small (since only one block out of arbitrarily many switches at each step). This has been observed in refs ${ }^{22,45}$, where typically the four-step switching paths have lower (but comparable) barriers than the two-step ones, which in turn have lower barriers than the single-step paths. (The path with a very large number of steps can be considered to be a simple model of domain wall in motion along the [001] direction.) However, this does not necessarily imply that the pathway with the highest number of steps determines the coercive field, because what is more important for the switching under an electric field is the slope of the energy vs. polarization curve ${ }^{42}$. For simplicity, as well as computational manageability, we focus only on two-step switching pathways.

Each of the four pathways can be reproduced within the same doubled conventional cell as the polar structure. The $P n^{*} a b$ and $\mathrm{Pn}^{*}$ am pathways (Fig. $7 \mathrm{a}, \mathrm{b}$ ) involve changes in the direction of the OOR mode, and both of them have nonpolar intermediate structures, with space groups Pnab and Pnam, respectively. The out-of-phase displacements of the A-site cations are along the polar axis in both of these intermediate structures. The $P b^{*} n m$ pathway involves switching the direction of the OOT mode $\left(X_{3}^{-}\right)$, whereas in the $P n^{*} 2_{1}^{*} m$ both OOR and OOT change directions, as shown in Fig. 7c, d. Mode decompositions of these switching pathways are given in the Supplementary information.

In Fig. 8a, we plot the energy as a function of the reaction coordinate for these four switching pathways in unstrained $\mathrm{Sr}_{3} \mathrm{Sn}_{2} \mathrm{O}_{7}$. The energy barriers are comparable and the lowest one is for the $\mathrm{Pn}^{*}$ am pathway. Results presented in Fig. 8b show how the energetics of this path behaves under tensile strain: Tensile strain monotonically decreases the $P n^{*} a m$ switching barrier, thus lowering the expected coercive field required for switching. This is not a surprising result, since the OOR's weaken under tensile strain, as shown in Fig. $6 \mathrm{C}$ and the $P n^{*}$ am pathway involves a change in the OOR character. What is interesting, and important for applications, is that this reduction in the switching barrier is not accompanied with a lower polarization under tensile 
(a) $P n^{*} a b$

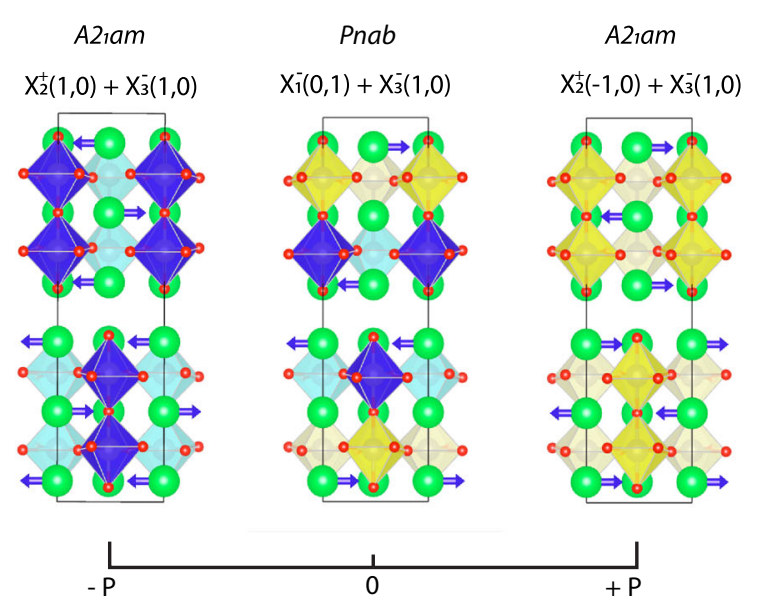

(c) $P b^{*} n m$

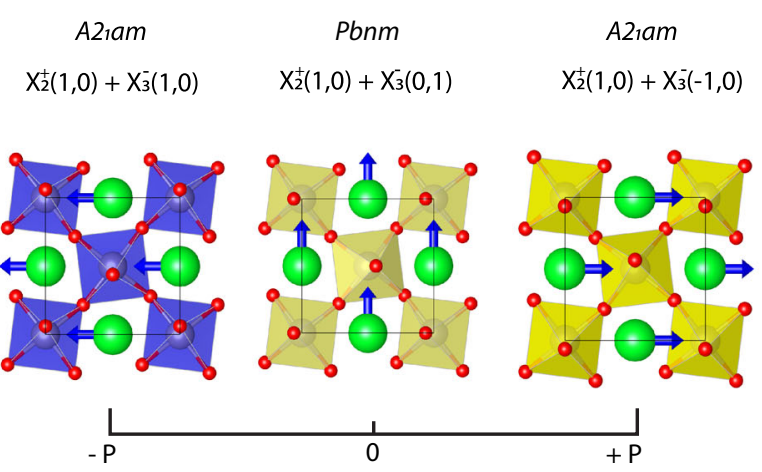

(b) $P n^{*} a m$

A21am
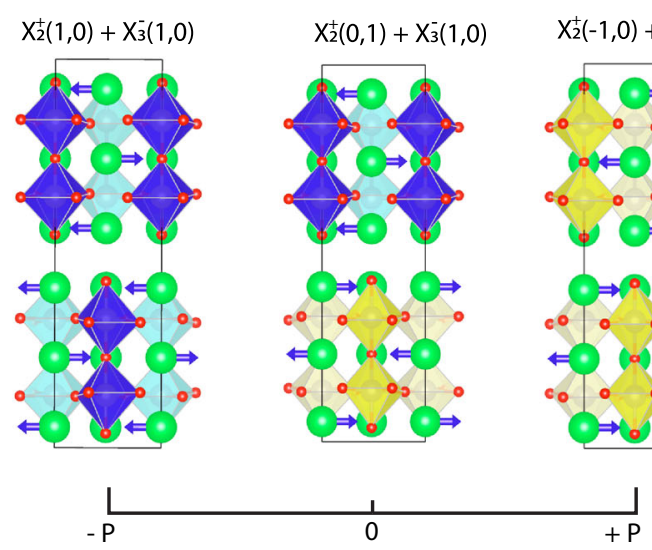

(d) $P n^{*} 21^{*} m$

$\begin{array}{ccc}\text { A2 1am } & \text { Bb21m } & \text { A2 1am } \\ \mathrm{X}_{2}^{ \pm}(1,0)+\mathrm{X}_{3}^{-}(1,0) & \mathrm{X}_{2}^{ \pm}(0,1)+\mathrm{X}_{3}(0,1) & \mathrm{X}_{2}^{ \pm}(1,0)+\mathrm{X}_{3}^{-}(-1,0)\end{array}$
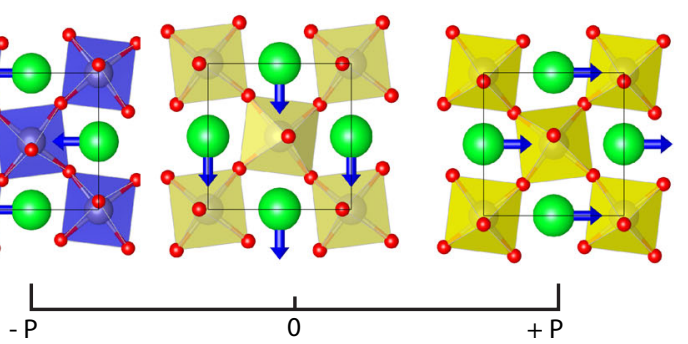

Fig. 7 Four possible polarization switching pathways. a $P n^{*} a b, \mathbf{b} P n^{*} a m, \mathbf{c} P b^{*} n m$, and $\mathbf{d} P n^{*} 2_{1}^{*} m$. The octahedra that remain in their original rotation direction are shown in blue, whereas those that switch their rotation direction are shown in yellow.
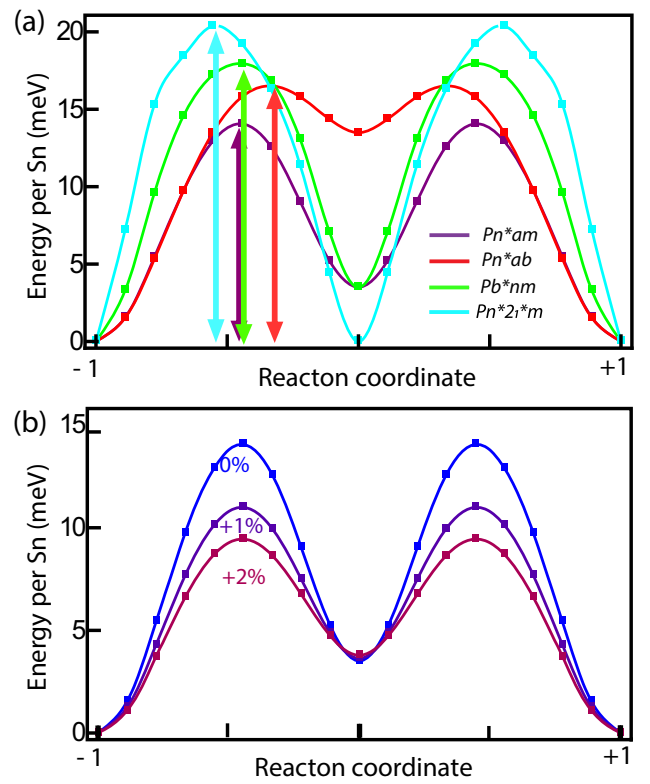

Fig. 8 Energy barriers for polarization switching in $\mathrm{Sr}_{3} \mathrm{Sn}_{2} \mathrm{O}_{7 .}$ a The energy barriers of different pathways for unstrained $\mathrm{Sr}_{3} \mathrm{Sn}_{2} \mathrm{O}_{7}$. The horizontal axis is the "reaction coordinate" that parametrizes the switching path. Arrows indicate the barrier heights. $\mathbf{b}$ The energy of the $\mathrm{Pn}^{*}$ am pathway in $\mathrm{Sr}_{3} \mathrm{Sn}_{2} \mathrm{O}_{7}$, as a function of tensile biaxial strain. strain (Fig. 4). Thus, strain can be used as a means to lower the coercive field of hybrid improper ferroelectrics.

The strong strain dependence of the switching barrier is not specific only to $\mathrm{Sr}_{3} \mathrm{Sn}_{2} \mathrm{O}_{7}$, or the $\mathrm{Pn}^{*}$ am pathway. In Fig. 9a, b, we show the barrier for different switching paths of $\mathrm{Sr}_{3} \mathrm{Sn}_{2} \mathrm{O}_{7}$ and $\mathrm{Ca}_{3} \mathrm{Ti}_{2} \mathrm{O}_{7}$ as a function of strain throughout the strain range that the HIF phase is stable. While the error bars in the energy barriers from the NEB calculations cause the curves to be rather rugged, two trends are evident: (i) under tensile strain, the barriers for pathways that involve changing the direction of the OOR mode $\left(P n^{*} a m\right.$ and $\left.P n^{*} a b\right)$ are lowered, and (ii) under compressive strain, the barrier for the pathway that only involve changing the direction of the OOT mode $\left(P b^{*} n m\right)$ is lowered. These are consistent with the tendencies towards OOR and OOT distortions becoming weaker under tensile and compressive strain as discussed earlier. Near $0 \%$ strain, the lowest barrier pathway switches from $P b^{*} n m$ or $P n^{*} 2_{1}^{*} m$ to either $P n^{*} a b$ or $P n^{*} a m$, and either strain direction leads to a lower coherent switching energy barrier. The lowest barriers are obtained near the phase boundaries between the polar and nonpolar phases, and the maximum suppression is about $50 \%$ in both compounds.

$\mathrm{Ca}_{3} \mathrm{Sn}_{2} \mathrm{O}_{7}$ has a lower tolerance factor than $\mathrm{Sr}_{3} \mathrm{Sn}_{2} \mathrm{O}_{7}$ and $\mathrm{Ca}_{3} \mathrm{Ti}_{2} \mathrm{O}_{7}$, and it does not display a strain-induced phase transition in the strain range we considered. It does not show a straininduced change in the switching pathway, or a significant decrease in the switching barrier either (Fig. 9c). This is likely because this compound is very far from the phase boundaries, and 
(a) $\mathrm{Sr}_{3} \mathrm{Sn}_{2} \mathrm{O}_{7}$

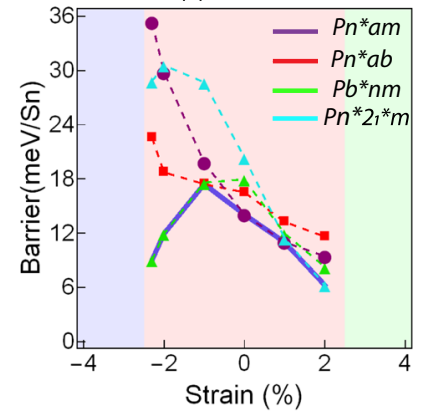

(b) $\mathrm{Ca}_{3} \mathrm{Ti}_{2} \mathrm{O}_{7}$

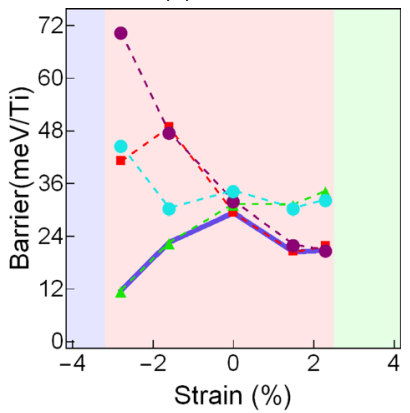

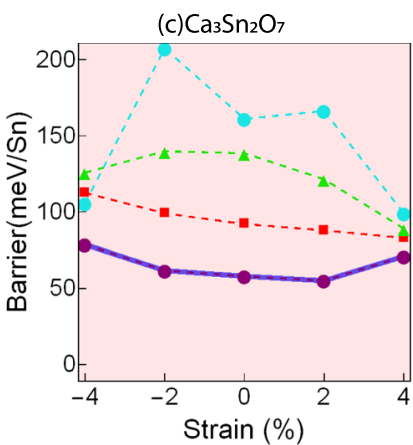

Fig. 9 The polarization switching barrier per B-site atom for (a) $\mathrm{Sr}_{3} \mathbf{S n}_{2} \mathbf{O}_{7}$, (b) $\mathbf{C a}_{3} \mathrm{Ti}_{2} \mathbf{O}_{7}$ and (c) $\mathrm{Ca}_{3} \mathbf{S n}_{2} \mathbf{O}_{7}$. The barriers for three distinct pathways are shown here, whilst the thick blue line is the minimum among those three. Background colors indicate different ground states.

with its small tolerance factor, it has such large OOR and OOT that the strain-induced changes in the instabilities are inconsequential.

\section{DISCUSSION}

Since its discovery about a decade ago, HIF have provided fertile ground for first principles materials by design approaches. Experiments have also been been catching up rapidly, verifying theoretical predictions. Multiple hybrid improper ferroelectric Ruddlesden-Popper phases have already been synthesized using bulk methods (for example, see refs ${ }^{4-6,11}$ ). Although thin film growth of Ruddlesden-Popper phases, especially for thermodynamically unstable compositions and at large strain values, is usually challenging because of the required stoichiometry control, there has been successful demonstration of switchable HIF in PLD grown films ${ }^{16}$, and both hybrid and conventional oxide molecular beam epitaxy have been used to synthesize phases that are not thermodynamically stable ${ }^{46,47}$. Current efforts focus on understanding more than the emergence of ferroelectricity, and to find ways to optimize properties such as the coercive field required for polarization switching.

In this study, we used first principles calculations to shed light on the strain-tolerance factor phase diagram of $n=2$ Ruddlesden-Popper HIF's, and to come up with a design strategy for obtaining lower coherent switching energy barriers. This quantity, which we used as a proxy for the coercive field, decreases significantly when strain is used to tune the HIF's to the nonpolar phase boundaries, because of the weakening of one of the rotation or tilt modes. We further showed that this weakening, and the resulting decrease in the switching barrier, is not always accompanied with a decrease in the polarization magnitude, for example in $\mathrm{Sr}_{3} \mathrm{Sn}_{2} \mathrm{O}_{7}$, verifying the point made early on in ref. ${ }^{9}$ that a lower barrier does not necessarily mean a lower polarization. Our results thus show that biaxial strain, which has historically been used to induce ferroelectricity in many oxides, can also be used as a means to tune the coercive field of hybrid improper ferroelectrics.

\section{METHODS}

First principles and other calculations

DFT calculations are performed using the projector-augmented wave approach $^{48}$ as implemented in the Vienna ab-initio simulation package (VASP) $^{49,50}$, and using the PBEsol generalized gradient approximation ${ }^{51}$. All calculations are done in a 48-atom (four formula unit) supercell, which can be viewed as a $\sqrt{2} \times \sqrt{2} \times 2$ multiple of the primitive cell of the reference $14 / \mathrm{mmm}$ structure. A $\Gamma$-centered $6 \times 6 \times 2$ grid of $\mathrm{k}$-points is used for the Brillouin zone integrals.

We consider all $\mathrm{A}_{3} \mathrm{~B}_{2} \mathrm{O}_{7}$ compounds with $\mathrm{A}=\mathrm{Ca}, \mathrm{Sr}, \mathrm{Ba}$ and $\mathrm{B}=\mathrm{Ti}, \mathrm{Zr}, \mathrm{Sn}$, $\mathrm{Ge}$, as well as $\mathrm{Cd}_{3} \mathrm{Ti}_{2} \mathrm{O}_{7}{ }^{4-6,33,52-55}$. These compounds are all band insulators with sizable gaps, so using the PBEsol generalized gradient approximation is expected to reproduce the crystal structures with reasonable accuracy. Biaxial strain boundary conditions are simulated by fixing the in-plane lattice constants, and allowing the out of plane component, as well as internal atomic positions, to relax with an force threshold of $2 \mathrm{meV} / \AA \AA$. The zero strain is defined for each compound by the a lattice constant obtained by completely relaxing the structure in the reference highsymmetry structure $14 / \mathrm{mmm}$.

The Goldschmidt tolerance factor ${ }^{56}$, which is used as a simple measure to predict tendency towards octahedral rotations, and is originally defined in terms of the ionic radii $r$ using

$$
\tau=\frac{r_{\mathrm{A}}+r_{\mathrm{O}}}{\sqrt{2}\left(r_{\mathrm{B}}+r_{\mathrm{O}}\right)}
$$

is instead calculated using the bond lengths for 12 coordinated A-site $\left(d_{\mathrm{AO}}\right)$ and 6 coordinated B-site $\left(d_{\mathrm{BO}}\right)$ ions from the bond valence model as

$\tau=\frac{d_{\mathrm{AO}}}{\sqrt{2} \cdot d_{\mathrm{BO}}}$.

(This approach is following ref. ${ }^{29}$.)

In order to calculate the minimum energy barrier for polarization switching, climbing-image nudged elastic band (Cl-NEB) method was used to further relax linearly interpolated switching paths to the minimum energy path ${ }^{57}$. The spring constant was set to $5 \mathrm{eV} / \AA^{2}$, and a convergence criterion of $1 \mathrm{meV}$ per supercell was used. Distortion symmetry groups ${ }^{44,58}$ are used to enumerate and name the possible initial pathways following ref. ${ }^{22}$ with the help of the DiSPy package ${ }^{59}$. All the switching pathways reported in the text retain their symmetry for all values of the reaction coordinate under NEB calculation.

As various points in this paper, symmetry, and group theory-related arguments are built using the Isotropy Software Package ${ }^{60}$ and the Bilbao Crystallographic Server ${ }^{61-63}$. VESTA software was used for visualization of crystal structures. ${ }^{64}$

\section{DATA AVAILABILITY}

Data for the phase diagram and the switching paths are available at the Data Repository for University of Minnesota at https://doi.org/10.13020/hvr3-bg02.

Received: 28 May 2020; Accepted: 11 October 2020; Published online: 06 November 2020

\section{REFERENCES}

1. Benedek, N. A. \& Fennie, C. J. Why are there so few perovskite ferroelectrics? J. Phys. Chem. C 117, 13339-13349 (2013).

2. Benedek, N. A. \& Fennie, C. J. Hybrid improper ferroelectricity: a mechanism for controllable polarization-magnetization coupling. Phys. Rev. Lett. 106, 107204 (2011).

3. Oh, Y. S., Luo, X., Huang, F.-T., Wang, Y. \& Cheong, S.-W. Experimental demonstration of hybrid improper ferroelectricity and the presence of abundant charged walls in (Ca,Sr)3Ti2O7 crystals. Nat. Mater. 14, 407-413 (2015).

4. Wang, Y., Huang, F. T., Luo, X., Gao, B. \& Cheong, S. W. The first room-temperature ferroelectric $\mathrm{Sn}$ insulator and its polarization switching kinetics. Adv. Mater. 29, 1601288 (2017). 
5. Yoshida, S. et al. Hybrid improper ferroelectricity in $(\mathrm{Sr}, \mathrm{Ca}) 3 \mathrm{Sn} 2 \mathrm{O} 7$ and beyond: universal relationship between ferroelectric transition temperature and tolerance factor in $n=2$ Ruddlesden-Popper phases. J. Am. Chem. Soc. 140, 15690-15700 (2018).

6. Yoshida, S. et al. Ferroelectric Sr3Zr2O7: competition between hybrid improper ferroelectric and antiferroelectric mechanisms. Adv. Funct. Mater. 28, 1801856 (2018).

7. Pitcher, M. J. et al. Tilt engineering of spontaneous polarization and magnetization above $300 \mathrm{~K}$ in a bulk layered perovskite. Science 347, 420-424 (2015).

8. Elcombe, M. M. et al. Structure determinations for Ca3Ti2O7, Ca4Ti3O10, Ca3.6Sr0.4Ti3O10 and a refinement of Sr3Ti2O7. Acta Crystallogr. B 47, 305-314 (1991).

9. Mulder, A. T., Benedek, N. A., Rondinelli, J. M. \& Fennie, C. J. Turning $\mathrm{ABO}$ antiferroelectrics into ferroelectrics: design rules for practical rotation-driven ferroelectricity in double perovskites and A3B2O7 Ruddlesden-Popper compounds. Adv. Funct. Mater. 23, 4810-4820 (2013).

10. Lines, M. \& Glass, A. Principles and Applications of Ferroelectrics and Related Materials. International Series of Monographs on Physics (OUP Oxford, 2001).

11. $\mathrm{Xu}, \mathrm{X}$. et al. Highly tunable ferroelectricity in hybrid improper ferroelectric Sr3Sn2O7. Adv. Funct. Mater. 2003623, 1-9 (2020)

12. Scott, J. F. Applications of modern ferroelectrics. Science 315, 954-959 (2007).

13. Cole, J., Ahmed, S. J., Curiel, L., Pichardo, S. \& Rubel, O. Marble game with optimal ferroelectric switching. J. Phys. Condens. Matter 26, 135901 (2014).

14. Xu, R. et al. Reducing coercive-field scaling in ferroelectric thin films via orientation control. ACS Nano 12, 4736-4743 (2018).

15. Liu, Z. Q. et al. Electrically reversible cracks in an intermetallic film controlled by an electric field. Nat. Commun. 9, 1-7 (2018).

16. $\mathrm{Li}, \mathrm{X}$. et al. Ultra-low coercive field of improper ferroelectric Ca3Ti2O7 epitaxial thin films. Appl. Phys. Lett. 110, 42901 (2017)

17. Wang, T. et al. Engineering $\mathrm{SrSnO} 3$ phases and electron mobility at room temperature using epitaxial strain. ACS Appl. Mater. Interfaces 10, 43802-43808 (2018).

18. Chaturvedi, V. et al. Strain-induced majority carrier inversion in ferromagnetic epitaxial $\mathrm{LaCoO}_{3-\delta}$ thin films. Phys. Rev. Mater. 4, 034403 (2020).

19. Clima, S. et al. Identification of the ferroelectric switching process and dopantdependent switching properties in orthorhombic HfO2: a first principles insight. Appl. Phys. Lett. 104, 092906 (2014).

20. Lu, X. Z. \& Rondinelli, J. M. Epitaxial-strain-induced polar-to-nonpolar transitions in layered oxides. Nat. Mater. 15, 951-955 (2016).

21. Nowadnick, E. A. \& Fennie, C. J. Domains and ferroelectric switching pathways in Ca3Ti2O7 from first principles. Phys. Rev. B 94, 104105 (2016).

22. Munro, J. M. et al. Discovering minimum energy pathways via distortion symmetry groups. Phys. Rev. B 98, 85107 (2018)

23. Ruddlesden, S. N. \& Popper, P. New compounds of the K2NiF4 type. Acta Crystallogr. 10, 538-539 (1957).

24. Ruddlesden, S. N. \& Popper, P. The compound Sr3Ti2O7 and its structure. Acto Crystallogr. 11, 54-55 (1958).

25. Birol, T., Benedek, N. A. \& Fennie, C. J. Interface control of emergent ferroic order in Ruddlesden-Popper Srn+1TinO3n+1. Phys. Rev. Lett. 107, 257602 (2011).

26. Zhang, H., Haule, K. \& Vanderbilt, D. Effective $J=1 / 2$ insulating state in Ruddlesden-Popper iridates: an LDA+DMFT study. Phys. Rev. Lett. 111, 246402 (2013).

27. Wang, Q. et al. Dimensionality-controlled mott transition and correlation effects in single-layer and bilayer perovskite iridates. Phys. Rev. B 87, 245109 (2013).

28. Li, W. et al. Band gap evolution in Ruddlesden-Popper phases. Phys. Rev. Mater. 3 , 101601 (2019).

29. Lufaso, M. W. \& Woodward, P. M. Prediction of the crystal structures of perovskites using the software program SPuDS. Acta Crystallogr. B 57, 725-738 (2001).

30. Miller, S. C. \& Love, W. F.Tables of Irreducible Representations of Space Groups and Co-representations of Magnetic Space Groups (Pruett Press, 1967).

31. Woodward, P. M. Octahedral tilting in perovskites. II. Structure stabilizing forces. Acta Crystallogr. B 53, 44-66 (1997).

32. Bradley, C. \& Cracknell, A.The Mathematical Theory of Symmetry in Solids: Representation Theory for Point Groups and Space Groups. EBSCO ebook Academic Collection (OUP Oxford, 2010).

33. Li, C. F. et al. Structural transitions in hybrid improper ferroelectric Ca3Ti2O7 tuned by site-selective isovalent substitutions: a first-principles study. Phys. Rev. $B$ 97 (2018).

34. Lu, X. Z. \& Rondinelli, J. M. Room temperature electric-field control of magnetism in layered oxides with cation order. Adv. Funct. Mater. 27, 1604312 (2017).

35. Salinas-Sanchez, A., Garcia-Muñoz, J. L., Rodriguez-Carvajal, J., Saez-Puche, R. \& Martinez, J. L. Structural characterization of R2BaCuO5 ( $R=Y, \mathrm{Lu}, \mathrm{Yb}, \mathrm{Tm}, \mathrm{Er}, \mathrm{Ho}$ Dy, Gd, Eu and Sm) oxides by X-ray and neutron diffraction. J. Solid State Chem. 100, 201-211 (1992).
36. Ghosez, P., Gonze, X. \& Michenaud, J.-P. Coulomb interaction and ferroelectric instability of BaTiO3. Europhys. Lett. 33, 713 (1996).

37. Yang, Y., Ren, W., Wang, D. \& Bellaiche, L. Understanding and revisiting properties of EuTiO3 bulk material and films from first principles. Phys. Rev. Lett. 109, 267602 (2012).

38. Zayak, A. T., Huang, X., Neaton, J. B. \& Rabe, K. M. Structural, electronic, and magnetic properties of SrRuO3 under epitaxial strain. Phys. Rev. B 74, 094104 (2006).

39. Zeches, R. J. et al. A strain-driven morphotropic phase boundary in BiFeO3. Science 326, 977-980 (2009).

40. Newnham, R. E. Phase transformations in smart materials. Acta Crystallogr. A 54, 729-737 (1998).

41. Birol, T. et al. The magnetoelectric effect in transition metal oxides: Insights and the rational design of new materials from first principles. Curr. Opin. Solid State Mater. Sci. 16, 227-242 (2012).

42. Beckman, S. P., Wang, X., Rabe, K. M. \& Vanderbilt, D. Ideal barriers to polarization reversal and domain-wall motion in strained ferroelectric thin films. Phys. Rev. $B$ 79, (2009).

43. Dittrich, R. et al. A path method for finding energy barriers and minimum energy paths in complex micromagnetic systems. J. Magn. Magn. Mater. 250, 12-19 (2002).

44. Vanleeuwen, B. K. \& Gopalan, V. The antisymmetry of distortions. Nat. Commun. 6 8818 (2015).

45. Liu, X. Q., Lu, J. J., Chen, B. H., Zhang, B. H. \& Chen, X. M. Hybrid improper ferroelectricity and possible ferroelectric switching paths in $\mathrm{Sr} 3 \mathrm{Hf} 2 \mathrm{O} 7$. J. Appl. Phys. 125, 114105 (2019).

46. Lee, C.-H. H. et al. Exploiting dimensionality and defect mitigation to create tunable microwave dielectrics. Nature 502, 532-536 (2013).

47. Haislmaier, R. C., Stone, G., Alem, N. \& Engel-Herbert, R. Creating Ruddlesden-Popper phases by hybrid molecular beam epitaxy. Appl. Phys. Lett. 109, 043102 (2016).

48. Blöchl, P. E. Projector augmented-wave method. Phys. Rev. B 50, 17953 (1994).

49. Kresse, G. \& Joubert, D. From ultrasoft pseudopotentials to the projector augmented-wave method. Phys. Rev. B 59, 1758-1775 (1999).

50. Kresse, G. \& Hafner, J. Ab initio molecular dynamics for liquid metals. Phys. Rev. $B$ 47, 558-561 (1993).

51. Perdew, J. P. et al. Restoring the density-gradient expansion for exchange in solids and surfaces. Phys. Rev. Lett. 100, 136406 (2008).

52. Lee, C.-H. et al. Exploiting dimensionality and defect mitigation to create tunable microwave dielectrics. Nature 502, 532 (2013).

53. Kennedy, B. J., Zhou, Q. \& Avdeev, M. The ferroelectric phase of CdTiO3: a powder neutron diffraction study. J. Solid State Chem. 184, 2987-2993 (2011).

54. Henriques, J., Caetano, E., Freire, V., da Costa, J. \& Albuquerque, E. Ab initio structural, electronic and optical properties of orthorhombic $\mathrm{CaGeO}$. J. Solid State Chem. 180, 974-980 (2007).

55. Moriwake, $\mathrm{H}$. et al. First-principles calculations of lattice dynamics in $\mathrm{CdTiO} 3$ and CaTiO3: phase stability and ferroelectricity. Phys. Rev. B 84, 104114 (2011).

56. Goldschmidt, V. M. Die gesetze der krystallochemie. Naturwissenschaften 14 477-485 (1926).

57. Henkelman, G. \& Jónsson, H. Improved tangent estimate in the nudged elastic band method for finding minimum energy paths and saddle points. J. Chem. Phys. 113, 9978-9985 (2000).

58. Padmanabhan, H., Munro, J. M., Dabo, I. \& Gopalan, V. Antisymmetry: fundamentals and applications. Annu. Rev. Mater. Res. 50, annurev-matsci-100219101404 (2020).

59. Munro, J. M., Liu, V. S., Gopalan, V. \& Dabo, I. Implementation of distortion symmetry for the nudged elastic band method with DiSPy. npj Comput. Mater. 5 52 (2019).

60. Stokes, H. T., Hatch, D. M. \& Campbell, B. J. ISOTROPY Software Suite, iso.byu.edu. (2007).

61. Aroyo, M. I., Kirov, A., Capillas, C., Perez-Mato, J. M. \& Wondratschek, H. Bilbao crystallographic server. II. Representations of crystallographic point groups and space groups. Acta Crystallogr. A 62, 115-128 (2006).

62. Aroyo, M. et al. Bilbao crystallographic server: I. Databases and crystallographic computing programs. Z. Kristallogr. 221, 15-27 (2006)

63. Aroyo, M. I. et al. Crystallography online: bilbao crystallographic server. Bulg. Chem. Commun. 43, 183-197 (2011).

64. Momma, K. \& Izumi, F. VESTA: a three-dimensional visualization system for electronic and structural analysis. J. Appl. Crystallogr. 41, 653-658 (2008).

65. Hatch, D. M. \& Stokes, H. T. INVARIANTS: program for obtaining a list of invariant polynomials of the order-parameter components associated with irreducible representations of a space group. J. Appl. Crystallogr. 36, 951-952 (2003) 


\section{ACKNOWLEDGEMENTS}

This work was supported primarily by the National Science Foundation through the University of Minnesota MRSEC under Award Number DMR-2011401. We acknowledge the Minnesota Supercomputing Institute (MSI) at the University of Minnesota for providing resources that contributed to the research results reported within this paper.

\section{AUTHOR CONTRIBUTIONS}

Both authors contributed to the planning of the project, and writing of the manuscript. First principles calculations were performed by S.L.

\section{COMPETING INTERESTS}

The authors declare no competing interests.

\section{ADDITIONAL INFORMATION}

Supplementary information is available for this paper at https://doi.org/10.1038/ s41524-020-00436-x.

Correspondence and requests for materials should be addressed to T.B.
Reprints and permission information is available at http://www.nature.com/ reprints

Publisher's note Springer Nature remains neutral with regard to jurisdictional claims in published maps and institutional affiliations.
cC) (i) Open Access This article is licensed under a Creative Commons cc) Attribution 4.0 International License, which permits use, sharing, adaptation, distribution and reproduction in any medium or format, as long as you give appropriate credit to the original author(s) and the source, provide a link to the Creative Commons license, and indicate if changes were made. The images or other third party material in this article are included in the article's Creative Commons license, unless indicated otherwise in a credit line to the material. If material is not included in the article's Creative Commons license and your intended use is not permitted by statutory regulation or exceeds the permitted use, you will need to obtain permission directly from the copyright holder. To view a copy of this license, visit http://creativecommons. org/licenses/by/4.0/.

(c) The Author(s) 2020 